\title{
IMPROVING THE QUALITY PROPERTIES OF COLD-SMOKED CATFISH (Clarias gariepinus L.) FILLETS TREATED WITH PHOSPHATE DURING COLD STORAGE
}

\author{
Waleed A. Abo El-Magd ${ }^{1}$, S.I. Ali ${ }^{1}$, S.E. El-Nemr ${ }^{2}$ and M. Ragab ${ }^{2}$ \\ 1. Cent. Lab. Aquac. Res., Agric. Res. Cent., Egypt \\ 2. Food Sci. Dept., Fac. Agric., Zagazig Univ., Egypt
}

\begin{abstract}
Phosphate has been successfully used in food processing. They are multi-purpose, generally recognized as safe (GRAS), and legally permitted additives to improve the quality of many foods. Therefore, the objective of this study was to assess effects of sodium tripolyphosphate (STPP) on the chemical, physical, microbiological and organoleptic quality attributes of cold-smoked catfish (Clarias gariepinus L.) fillets during storage at $4 \pm 1^{\circ} \mathrm{C}$ for 30 days. Results indicated that control samples have been shown to decrease colour, odour, taste and texture, and show a gradual deterioration in quality attributes with cold storage. The rates $(\mathrm{p}<0.05)$ of these deteriorations are increased as the time of cold storage progressed. On the other hand, STPP treated samples exhibit higher significance of $(p<0.05)$ moisture, protein and fat contents, at any given time of storage as compared with control samples. The present data also demonstrate lower significant values $(\mathrm{p}<0.05)$ of ash content, total volatile bases nitrogen (TVBN), trimethyl amine nitrogen (TMAN), thiobarbituric acid (TBA), pH-value, total bacterial, E. coli, moulds, yeasts, and psychrotrophic bacterial counts in phosphate treated samples. Panellist's evaluation indicated preference for STPP-treated samples when compared with control samples. Results indicated that chemical, physiochemical, microbiological and sensorial quality advantages have been resulted from soaked catfish fillets before smoking in different levels of sodium tripolyphosphate solution for $10 \mathrm{~min}$. It could be concluded that, catfish fillets treated with $5 \%$ sodium tripolyphosphate (STPP) for 10 min., cold smoked and packaged in polyethylene bags an alternative way to improve the quality of cold-smoked catfish fillets during cold storage at $4 \pm 1^{\circ} \mathrm{C}$ for 30 days.
\end{abstract}

Key words: Catfish, smoked fillets, cold storage, phosphate.

\section{INTRODUCTION}

Catfish is popular fresh water fish with an economically important group of fresh and brackish water fish worldwide. Several species have been successfully introduced in aquaculture and the African catfish, (Clarias gariepinus L.) is perhaps the most important one, not only in Africa but also in Southeast Asia and in Europe (Olayemi et al., 2011). Catfish meat quality can be negatively affected by the activity and stress of harvest and transport (Bosworth et al., 2004).
Smoke curing is a traditional fish preservation method of considerable economic importance worldwide. Smoke, is produced by the process of incomplete combustion of wood. It consists of numerous individual components namely: aldehydes, ketones, alcohols, acids, hydrocarbons, esters, phenols, ethers, etc. (Guillen and Errecalde, 2002). These components are transferred to the smoked goods by deposition on their surface and subsequent penetration into their flesh. The temperature of the smoke is in the range of $12-25^{\circ} \mathrm{C}$ during cold smoking and $25-45^{\circ} \mathrm{C}$ in warm smoking. Smoking imparts a

\footnotetext{
*Corresponding author: Tel. : +201112005334

E-mail address: waleedaboelmagd2012@yahoo.com
} 
characteristic flavour and colour to the fish. In addition, smoking increases the shelf-life of fish as a result of the combined effects of dehydration, antimicrobial and antioxidant activity of several of the smoke constituents mainly: formaldehyde, carboxylic acids and phenols (Leroi and Joffraud, 2000).

Phosphate is an indispensable additive for the maintenance of the functional properties of the seafood proteins which helps the preservation of the muscle integrity, inhibits the drip loss and helps to prevent the quality loss during storage (Moawad et al., 2013). Sodium tripolyphosphate (STPP) enhanced the sensory perception of moistness in treated fish, because of its water retention ability. The effectiveness of phosphates on water-retention properties of meat products depends on the type and quantity of phosphate and the specific food product. Phosphates have broad spectrum antimicrobial activity and are used for improving the microbiological quality of muscle and to improve shelf-life of sea food products (Thorarinsdottir et al., 2004). Among the functional properties changed by the treatment of phosphates in seafood and its products are the retention of the moisture and natural flavour, inhibiting the loss of fluids during the processing and storage, enhancing tenderness of seafood by restricting protein denaturation and stabilization of the colour (Turan et al., 2003).

Due to catfish lower market price and its less quality attributes, it is not desirable for Egyptian consumers. Therefore this work was carried out to study the utilization of catfish (Clarias gariepinus L.) as new untraditional source for the production of smoked fillets treated with different concentrations of sodium tripolyphosphate (STPP) by cold- smoking and studys the effect of storage at $4 \pm 1^{\circ} \mathrm{C}$ for 30 day on chemical, physical, microbiological and sensory characteristics.

\section{MATERIALS AND METHODS}

\section{Fish Samples}

Catfish (Clarias gariepinus L.) body weight ranged from (1500-2000 g) for each was obtained from Central Laboratory for Aquaculture Research, Abbassa, Abou-Hammad District,
Sharkia Governorate, Egypt, then transferred directly to the fish processing and quality control laboratory.

\section{Preparation of Fish Fillets}

About $20 \mathrm{~kg}$ of fresh catfish were slaughtered just below the head then left 15 minutes for bleeding. The fish washed by tap water to remove slime, darts, debris and residual blood on fish surface. Thereafter, skinning and filleting were carefully performed to avoid fillets contamination; the fillets washed by distilled water and kept under cooling (less than $4 \pm 1^{\circ} \mathrm{C}$ ). The percentage of fillets in catfish was $47.25 \%$ and average weight of fillets was about $400 \mathrm{~g}$.

\section{Discolouration of Catfish Fillets (Lighter Colour)}

First step: fillets washed with submerged in $0.2 \% \mathrm{NaHCO}_{3}$ solution 1:3 (W/V) for $10 \mathrm{~min}$. Second step: fillets washed by distilled water only 1:3 (W/V) for $10 \mathrm{~min}$. Last step: fillets washed with submerged in $0.5 \% \mathrm{NaCl}$ solution 1:3 (W/V) for $10 \mathrm{~min}$. as described by Atef et al. (2008).

\section{Preparation and Treatment Using Sodium Tripolyphosphate Solutions}

Phosphate solution was prepared by dissolving phosphate powder in distilled water at concentrations of 1,3 and $5 \%$ phosphate/liter water.

Catfish fillets were divided into four portions, one portion was untreated and served as control. The other three portions were immersed in phosphate solutions containing 1,3 and $5 \%$ phosphate/liter for $10 \mathrm{~min}$. at room temperature as described by Moawad et al. (2013). The ratio of fish to phosphate solution was 1:1(W/V).

\section{Salting and Smoking of Fillets Samples}

All catfish fillets were immersed in a brine containing $15 \% \mathrm{NaCl}$ at room temperature for two hours with a fish: brine ratio of 1:1 (W/V). Then the fillets were washed with tap water and kept on net shelves and allowed to drain overnight inside a chill room.

The previous salted fish fillets were smoked at Misr-Aswan Company for Fishing and Fish Processing, at Tenth Ramadan City, Egypt. The 
cold- smoking process included three stages as described by Sulieman (1993) and it was employed as follows:

1. A preliminary drying stage with air at $30^{\circ} \mathrm{C}$ for $30 \mathrm{~min}$.

2. A smoking stage at $30^{\circ} \mathrm{C}$. for 4 hours using Sweden sawdust wood, which was moistened with water to increase the moisture content to $30 \%$ as source of smoke.

3. A heating stage at $50^{\circ} \mathrm{C}$ without smoke for one hour.

About $9.5 \mathrm{~kg}$ fillets were packaged in polyethylene bags of $0.15 \mathrm{~mm}$ thickness (Made in Egypt). A half kg of packaged smoked fillets were put in a foam plate and wrapped in cling film. All smoked samples were stored in a refrigerator at $4 \pm 1^{\circ} \mathrm{C}$ till analysis. Smoked samples were analyzed directly after smoking; and at intervals of 3 days during storage for 30 days for chemical, physical, microbiological and sensory evaluation.

\section{Analytical Methods}

The fish samples were minced by Braun mincer and well mixed for analysis. Moisture, total protein, ash, and total lipids were determined as described by AOAC (2005). Total volatile bases nitrogen (TVBN), and trimethylamine nitrogen (TMAN) were determined according to the method recommended by the Malle and Tao (1986).
Thiobarbituric acid (TBA) was assessed according to the method described by Tarladgis et al. (1960). pH was estimated according to the method mentioned by Özogul et al. (2005), using pH-meter (Orion pH-meter Model 420 A). Total bacterial count (TBC) and psychrophilic bacterial count (PsBC) were detected according to the method described by Swanson et al. (1992) using nutrient agar media. E. coli and mould and yeast counts were counted according to the method described in APHA (1992) using maconky agar media for E. coli and potato dextrose agar media for mould and yeast counts.

Organoleptic properties were evaluated for colour, odour, taste and texture during storage periods. Group of 9 judges from Central Laboratory for Aquaculture Research, Abbassa, Abou-Hammad District, Sharkia, Egypt were always called upon for scoring the organoleptic properties of the samples by given grads ranging from 0 to 10 as described by Teeny and Miyauchi (1972) as shown in Table 1.

\section{Statistical Analysis}

The obtained data of different parameters were statistically analyzed using SPSS software (14.0 for Windows) (Statsoft, 1994). Data was expressed as mean \pm SD and were analyzed by one-way ANOVA test. Duncan's test (1955) was used to test the differences between means. The significance level was $\mathrm{P}<0.05$.

Table 1. Description of organoleptic properties scores

\begin{tabular}{llll}
\hline Score & Description & Score & Description \\
\hline 10 & Ideal & 4 & Fair \\
9 & Excellent & 3 & Poorly fair \\
8 & Very good & 2 & Poor \\
7 & Good & 1 & Very poor \\
6 & Fairly good & 0 & Repulsive \\
5 & Acceptable & & \\
\hline
\end{tabular}




\section{RESULTS AND DESCUSSION}

\section{Effect of Cold Storage on Chemical Composition and Physical Properties of Cold-Smoked Catfish Fillets Treated with Different Concentrations of Phosphate}

\section{Moisture content}

Table 2 indicates that moisture content slowly decreased in all studied samples, but the decrement of 5\% sodium tripolyphosphate (STPP) treated sample was less than others. While, the decrease was more in control, $1 \%$ and 3\% STPP treated samples, respectively. The moisture content of all samples gradually decreased with a higher rate $(\mathrm{P}<0.05)$ in control samples than in STPP treated samples. Moreover, at any given time of storage phosphate-treated samples showed higher moisture content than control samples, which indicated that STPP protected the smoked catfish fillets samples from dehydration during cold storage. The loss of moisture contents ranged from $2.3 \%$ to $4.78 \%$ after 30 days of cold storages; this may be associated with changes occurred in protein and lipid as well as the dehydration of the smoked fillets. The data are in agreement with those reported by Ibrahim (1999) Abd El-Halim (2003) and Egbal et al. (2013).

\section{Protein content}

Slight decrease in protein content was observed during cold storage of studied samples. The levels of protein content significantly decreased $(\mathrm{p}<0.05)$ during cold storage for all treatments (Table 2) particularly in control sample and 1\% STPP treated fillets. The decrease in 5\% STPP treated sample $(0.97 \%)$ was less than control, 1 and 3\% STPP treated samples which lost 1.59, 1.27 and $1.12 \%$ from its protein contents at the end of storage period, respectively. The decrease in protein content may be due to the enzymatic proteolysis which leads to the formation of simple nitrogenous compounds. The obtained results are in accordance with El-Akeel (1988), Saeed and Howell (2002) and Alcicek and Atar (2010).

\section{Fat content}

Data illustrated in Table 3 revealed that the levels of fat content significantly decreased $(\mathrm{P}<0.05)$ during cold storage for all treatments.
Fat content was $16.40 \%$ for all samples at zero time of storage period, while at the end of storage period was 14.93, 15.40, 15.71 and $15.89 \%$ for control, 1, 3 and 5\% STPP treated samples, respectively. The highest level in fat content was recorded in samples treated with phosphate particularly in case of 5\% STPP while, the lowest level was observed in control samples. So that, the case of 5\% STPP has the highest effect to prolong the storage period, followed by 3 and 1\% STPP. The decrease in fat content of cold-smoked catfish samples may be due to further hydrolysis and oxidation of lipids. The obtained results are in accordance with ElAkeel (1988) Saeed and Howell (2002) and Alcicek and Atar (2010).

\section{Ash content}

The effect of cold storage on ash content of smoked catfish fillets treated with phosphate is represented in Table 3. Ash content was significantly increased $(\mathrm{p}<0.05)$ during cold storage for all treatments. The ash content was increased gradually during storage. Ash content was $15.10 \%$ for all samples at zero time. After 30 days of storage, ash content values were $18.65,17.83,17.33$ and $16.98 \%$ for control, 1,3 and 5\% STPP samples, respectively. Data in this Table show that, the lowest level in ash content was recorded in samples treated with 5\% STPP while the highest level in ash content was observed in control samples. The increase in ash may be due to loss of moisture, protein and conversion of some lipids into aldehydes and ketones and breakdown of fatty acids. These results are in coincidence with those reported by Serkan et al. (2009), Kumolu-Johnson et al. (2010) and Egbal et al. (2013).

\section{pH-value}

The changes in the $\mathrm{pH}$ of smoked catfish fillets treated with phosphate are given in Table 4. The $\mathrm{pH}$ values, significantly increased $(\mathrm{p}<0.05)$ during cold storage for all treatments. $\mathrm{pH}$ values were $6.18,6.23,6.25$ and 6.28 for smoked catfish fillets control, 1, 3, and 5\% STPP, respectively at zero time. While, the $\mathrm{pH}$ values at the end of storage period after 30 days were $6.57,6.39,6.34$ and 6.21, respectively. This increase in $\mathrm{pH}$ value might be mainly due to the activity of some bacteria and activity of fish tissue enzymes on fish protein decomposition causing the formation of free amino 
Table 2. Changes in moisture and protein contents (\%) of cold-smoked catfish fillets treated with 1,3 and $5 \%$ sodium tripolyphosphate (STPP) during cold storage at $4 \pm 1^{\circ} \mathrm{C}$ for 30 days

\begin{tabular}{|c|c|c|c|c|c|c|c|c|}
\hline \multirow{3}{*}{$\begin{array}{l}\text { Parameter } \\
\text { Storage } \\
\text { period } \\
\text { (day) }\end{array}$} & \multicolumn{4}{|c|}{ Moisture } & \multicolumn{4}{|c|}{ Protein* } \\
\hline & \multicolumn{8}{|c|}{ STPP treatments } \\
\hline & Control & $1 \%$ STPP & $3 \%$ STPP & 5\% STPP & Control & $1 \%$ STPP & $3 \%$ STPP & $5 \%$ STPP \\
\hline \multirow[t]{2}{*}{$\mathbf{0}$} & $62.54 \pm$ & $62.54 \pm$ & $62.54 \pm$ & $62.54 \pm$ & $67.90 \pm$ & $67.90 \pm$ & $67.90 \pm$ & $67.90 \pm$ \\
\hline & $0.70^{\mathrm{a}}$ & $0.74^{\mathrm{a}}$ & $0.67^{\mathrm{a}}$ & $0.71^{\mathrm{a}}$ & $0.71^{\mathrm{a}}$ & $0.78 \mathrm{a}$ & 0.70a & $0.79 a$ \\
\hline \multirow[t]{2}{*}{3} & $62.16 \pm$ & $62.19 \pm$ & $62.25 \pm$ & $62.35 \pm$ & $67.72 \pm$ & $67.77 \pm$ & $67.78 \pm$ & $67.84 \pm$ \\
\hline & $0.64^{\mathrm{a}}$ & $0.68^{\mathrm{a}}$ & $0.54^{\mathrm{a}}$ & $0.74^{\mathrm{a}}$ & $0.68^{\mathrm{ab}}$ & $0.64 \mathrm{a}$ & $0.75 a$ & $0.74 \mathrm{a}$ \\
\hline \multirow[t]{2}{*}{6} & $61.65 \pm$ & $61.77 \pm$ & $61.91 \pm$ & $62.09 \pm$ & $67.60 \pm$ & $67.68 \pm$ & $67.73 \pm$ & $67.82 \pm$ \\
\hline & $0.66^{\mathrm{ab}}$ & $0.70^{\mathrm{ab}}$ & $0.62^{\mathrm{a}}$ & $0.70^{\mathrm{a}}$ & $0.64^{\mathrm{ab}}$ & $0.61^{\mathrm{ab}}$ & $0.64^{\mathrm{a}}$ & $0.70^{\mathrm{a}}$ \\
\hline \multirow[t]{2}{*}{9} & $61.25 \pm$ & $61.34 \pm$ & $61.54 \pm$ & $61.83 \pm$ & $67.38 \pm$ & $67.56 \pm$ & $67.60 \pm$ & $67.71 \pm$ \\
\hline & $0.78^{\mathrm{ab}}$ & $0.64^{\mathrm{ab}}$ & $0.39^{\mathrm{a}}$ & $0.54^{\mathrm{a}}$ & $0.74^{\mathrm{b}}$ & $0.65^{\mathrm{ab}}$ & $0.64^{\mathrm{ab}}$ & $0.64^{\mathrm{a}}$ \\
\hline \multirow[t]{2}{*}{12} & $60.67 \pm$ & $60.91 \pm$ & $61.23 \pm$ & $61.62 \pm$ & $67.21 \pm$ & $67.41 \pm$ & $67.48 \pm$ & $67.60 \pm$ \\
\hline & $0.63 b$ & $0.57^{\mathrm{ab}}$ & $0.66^{\mathrm{ab}}$ & $0.64^{\mathrm{a}}$ & $0.67^{\mathrm{b}}$ & $0.66^{\mathrm{b}}$ & $0.57^{\mathrm{ab}}$ & $0.54^{\mathrm{a}}$ \\
\hline \multirow[t]{2}{*}{15} & $60.20 \pm$ & $60.57 \pm$ & $60.89 \pm$ & $61.39 \pm$ & $67.11 \pm$ & $67.30 \pm$ & $67.37 \pm$ & $67.48 \pm$ \\
\hline & $0.70^{\mathrm{b}}$ & $0.64^{\mathrm{b}}$ & $0.54^{\mathrm{ab}}$ & $0.54^{\mathrm{a}}$ & $0.62^{\mathrm{b}}$ & $0.54^{\mathrm{ab}}$ & $0.67^{\mathrm{ab}}$ & $0.67^{\mathrm{a}}$ \\
\hline \multirow[t]{2}{*}{18} & $59.81 \pm$ & $60.18 \pm$ & $60.58 \pm$ & $61.17 \pm$ & $67.00 \pm$ & $67.17 \pm$ & $67.26 \pm$ & $67.39 \pm$ \\
\hline & $0.68^{\mathrm{bc}}$ & $0.64^{\mathrm{b}}$ & $0.58^{\mathrm{ab}}$ & $0.55^{\mathrm{a}}$ & $0.64^{\mathrm{b}}$ & $0.64^{\mathrm{b}}$ & $0.70^{\mathrm{ab}}$ & $0.70^{\mathrm{a}}$ \\
\hline \multirow[t]{2}{*}{21} & $59.31 \pm$ & $59.75 \pm$ & $60.26 \pm$ & $60.95 \pm$ & $66.83 \pm$ & $67.05 \pm$ & $67.14 \pm$ & $67.26 \pm$ \\
\hline & $0.70^{\mathrm{C}}$ & $0.76^{\mathrm{ab}}$ & $0.57^{\mathrm{a}}$ & $0.64^{\mathrm{a}}$ & $0.56^{\mathrm{bc}}$ & $0.58^{\mathrm{b}}$ & $0.64^{\mathrm{ab}}$ & $0.64^{\mathrm{a}}$ \\
\hline \multirow[t]{2}{*}{24} & $58.88 \pm$ & $59.34 \pm$ & $59.90 \pm$ & $60.70 \pm$ & $66.61 \pm$ & $66.92 \pm$ & $67.02 \pm$ & $67.17 \pm$ \\
\hline & $0.55^{\mathrm{c}}$ & $0.64^{\mathrm{bc}}$ & $0.69^{\mathrm{b}}$ & $0.70^{\mathrm{a}}$ & $0.60^{\mathrm{bc}}$ & $0.57^{\mathrm{b}}$ & $0.61^{\mathrm{ab}}$ & $0.61^{\mathrm{a}}$ \\
\hline \multirow[t]{2}{*}{27} & $58.14 \pm$ & $58.93 \pm$ & $59.52 \pm$ & $60.45 \pm$ & $66.48 \pm$ & $66.79 \pm$ & $66.91 \pm$ & $67.05 \pm$ \\
\hline & $0.58^{\mathrm{d}}$ & $0.60^{\mathrm{bc}}$ & $0.74^{\mathrm{b}}$ & $0.64^{\mathrm{a}}$ & $0.67^{\mathrm{c}}$ & $0.65^{\mathrm{b}}$ & $0.64^{\mathrm{b}}$ & $0.60^{\mathrm{a}}$ \\
\hline \multirow[t]{2}{*}{30} & $57.76 \pm$ & $58.58 \pm$ & $59.24 \pm$ & $60.20 \pm$ & $66.31 \pm$ & $66.63 \pm$ & $66.78 \pm$ & $66.93 \pm$ \\
\hline & $0.44^{\mathrm{d}}$ & $0.57^{\mathrm{c}}$ & $0.50^{\mathrm{b}}$ & $0.48^{\mathrm{a}}$ & $0.56^{\mathrm{c}}$ & $0.64^{\mathrm{bc}}$ & $0.54^{\mathrm{b}}$ & $0.58^{\mathrm{a}}$ \\
\hline
\end{tabular}

\footnotetext{
${ }^{\mathrm{a}-\mathrm{d}}$ Means within a raw with different superscript are significantly different $(\mathrm{P}<0.05)$.
}

Values are expressed as Mean \pm SD. $\quad *$ on dry weight bases. 
Table 3. Changes in fat and ash contents (\%) of cold-smoked catfish fillets treated with 1,3 and $5 \%$ sodium tripolyphosphate (STPP) during cold storage at $4 \pm 1^{\circ} \mathrm{C}$ for 30 days (on dry weight bases)

\begin{tabular}{|c|c|c|c|c|c|c|c|c|}
\hline \multirow{3}{*}{$\begin{array}{l}\text { Parameter } \\
\text { Storage } \\
\text { period } \\
\text { (day) }\end{array}$} & \multicolumn{4}{|c|}{ Fat } & \multicolumn{4}{|c|}{ Ash } \\
\hline & \multicolumn{8}{|c|}{ STPP treatments } \\
\hline & Control & 1\% STPP & 3\% STPP & $5 \%$ STPP & Control & $1 \%$ STPP & $3 \%$ STPP & $5 \%$ STPP \\
\hline \multirow[t]{2}{*}{$\mathbf{0}$} & $16.40 \pm$ & $16.40 \pm$ & $16.40 \pm$ & $16.40 \pm$ & $15.10 \pm$ & $15.10 \pm$ & $15.10 \pm$ & $15.10 \pm$ \\
\hline & $0.10^{\mathrm{a}}$ & $0.12^{\mathrm{a}}$ & $0.11 \mathrm{a}$ & $0.08^{\mathrm{a}}$ & $0.16 \mathrm{a}$ & $0.14 \mathrm{a}$ & $0.14 \mathrm{a}$ & 0.13a \\
\hline \multirow[t]{2}{*}{3} & $16.36 \pm$ & $16.32 \pm$ & $16.35 \pm$ & $16.35 \pm$ & $15.32 \pm$ & $15.31 \pm$ & $15.27 \pm$ & $15.22 \pm$ \\
\hline & $0.11^{\mathrm{a}}$ & $0.09^{\mathrm{a}}$ & $0.07 \mathrm{a}$ & $0.08^{\mathrm{a}}$ & $0.15^{\mathrm{a}}$ & $0.13^{\mathrm{a}}$ & $0.11^{\mathrm{a}}$ & $0.10^{\mathrm{ab}}$ \\
\hline \multirow[t]{2}{*}{6} & $16.15 \pm$ & $16.24 \pm$ & $16.27 \pm$ & $16.31 \pm$ & $15.66 \pm$ & $15.49 \pm$ & $15.41 \pm$ & $15.28 \pm$ \\
\hline & $0.10^{\mathrm{a}}$ & $0.12 \mathrm{a}$ & $0.08 a$ & $0.09^{\mathrm{a}}$ & $0.14^{\mathrm{a}}$ & $0.14^{\mathrm{ab}}$ & $0.13^{\mathrm{ab}}$ & $0.11^{\mathrm{b}}$ \\
\hline \multirow[t]{2}{*}{9} & $16.03 \pm$ & $16.15 \pm$ & $16.21 \pm$ & $16.24 \pm$ & $16.01 \pm$ & $15.71 \pm$ & $15.60 \pm$ & $15.47 \pm$ \\
\hline & 0.07ab & $0.09^{\mathrm{a}}$ & $0.08 \mathrm{a}$ & $0.10^{\mathrm{a}}$ & $0.13 a$ & $0.15^{\mathrm{ab}}$ & $0.15^{\mathrm{bc}}$ & $0.13^{\mathrm{bc}}$ \\
\hline \multirow[t]{2}{*}{12} & $15.82 \pm$ & $16.03 \pm$ & $16.13 \pm$ & $16.17 \pm$ & $16.40 \pm$ & $16.00 \pm$ & $15.81 \pm$ & $15.68 \pm$ \\
\hline & $0.09^{\mathrm{ab}}$ & $0.08^{\mathrm{ab}}$ & $0.10^{\mathrm{a}}$ & $0.08^{\mathrm{a}}$ & $0.11^{\mathrm{a}}$ & $0.15^{\mathrm{b}}$ & $0.14^{\mathrm{bc}}$ & $0.15^{\mathrm{bc}}$ \\
\hline \multirow[t]{2}{*}{15} & $15.65 \pm$ & $15.94 \pm$ & $16.07 \pm$ & $16.10 \pm$ & $16.72 \pm$ & $16.23 \pm$ & $15.99 \pm$ & $15.92 \pm$ \\
\hline & $0.10^{\mathrm{b}}$ & $0.10^{\mathrm{ab}}$ & $0.08^{\mathrm{ab}}$ & $0.11^{\mathrm{a}}$ & $0.16^{\mathrm{a}}$ & $0.16^{\mathrm{b}}$ & $0.15^{\mathrm{bc}}$ & $0.16 \mathrm{c}$ \\
\hline \multirow[t]{2}{*}{18} & $15.56 \pm$ & $15.83 \pm$ & $16.00 \pm$ & $16.05 \pm$ & $16.97 \pm$ & $16.52 \pm$ & $16.23 \pm$ & $16.12 \pm$ \\
\hline & $0.09 \mathrm{~b}$ & $0.09^{\mathrm{b}}$ & $0.06^{\mathrm{ab}}$ & $0.08^{\mathrm{a}}$ & $0.13^{\mathrm{a}}$ & $0.17^{\mathrm{b}}$ & $0.16^{\mathrm{c}}$ & $0.12^{\mathrm{c}}$ \\
\hline \multirow[t]{2}{*}{21} & $15.38 \pm$ & $15.75 \pm$ & $15.92 \pm$ & $15.97 \pm$ & $17.38 \pm$ & $16.77 \pm$ & $16.51 \pm$ & $16.37 \pm$ \\
\hline & $0.06^{\mathrm{c}}$ & $0.08^{\mathrm{b}}$ & $0.09^{\mathrm{ab}}$ & $0.10^{\mathrm{a}}$ & $0.15^{\mathrm{a}}$ & $0.14^{\mathrm{b}}$ & $0.14^{\mathrm{c}}$ & $0.14^{\mathrm{cd}}$ \\
\hline \multirow[t]{2}{*}{24} & $15.23 \pm$ & $15.63 \pm$ & $15.84 \pm$ & $15.90 \pm$ & $17.81 \pm$ & $16.06 \pm$ & $16.78 \pm$ & $16.57 \pm$ \\
\hline & $0.11 \mathrm{c}$ & $0.10^{\mathrm{b}}$ & $0.08^{\mathrm{ab}}$ & $0.09^{\mathrm{a}}$ & $0.16^{\mathrm{a}}$ & $0.15^{\mathrm{b}}$ & $0.17^{\mathrm{c}}$ & $0.16^{\mathrm{cd}}$ \\
\hline \multirow[t]{2}{*}{27} & $15.06 \pm$ & $15.51 \pm$ & $15.78 \pm$ & $15.83 \pm$ & $18.21 \pm$ & $16.40 \pm$ & $17.06 \pm$ & $16.83 \pm$ \\
\hline & $0.07^{\mathrm{c}}$ & $0.11^{\mathrm{bc}}$ & 0.08 & $0.08^{\mathrm{a}}$ & $0.18^{\mathrm{a}}$ & $0.18^{\mathrm{b}}$ & $0.18^{\mathrm{c}}$ & $0.16^{\mathrm{d}}$ \\
\hline \multirow[t]{2}{*}{30} & $14.93 \pm$ & $15.40 \pm$ & $15.71 \pm$ & $15.89 \pm$ & $18.65 \pm$ & $17.83 \pm$ & $17.33 \pm$ & $16.98 \pm$ \\
\hline & $0.07^{\mathrm{d}}$ & $0.08^{\mathrm{bc}}$ & & $0.07^{\mathrm{a}}$ & $0.18^{\mathrm{a}}$ & $0.16^{\mathrm{b}}$ & $0.15^{\mathrm{c}}$ & $0.15^{\mathrm{d}}$ \\
\hline
\end{tabular}

${ }^{\mathrm{a}-\mathrm{d}}$ Means within a raw with different superscript are significantly different $(\mathrm{P}<0.05)$.

Values are expressed as Mean \pm SD. 
Table 4. Changes in pH values and thiobarbituric acid (TBA) contents (mg MDA/kg) of coldsmoked catfish fillets treated with 1, 3 and $5 \%$ sodium tripolyphosphate (STPP) during cold storage at $4 \pm 1^{\circ} \mathrm{C}$ for 30 days (on wet weight bases)

\begin{tabular}{|c|c|c|c|c|c|c|c|c|}
\hline \multirow{3}{*}{$\begin{array}{l}\text { Parameter } \\
\text { Storage } \\
\text { period } \\
\text { (day) }\end{array}$} & \multicolumn{4}{|c|}{ pH } & \multicolumn{4}{|c|}{ TBA (mg MDA/kg) } \\
\hline & \multicolumn{8}{|c|}{ STPP treatments } \\
\hline & Control & 1\% STPP & 3\% STPP & $5 \%$ STPP & Control & $1 \%$ STPP & $3 \%$ STPP & $5 \%$ STPP \\
\hline \multirow[t]{2}{*}{0} & $6.18 \pm$ & $6.23 \pm$ & $6.25 \pm$ & $6.28 \pm$ & $0.75 \pm$ & $0.73 \pm$ & $0.73 \pm$ & $0.71 \pm$ \\
\hline & $0.02^{\mathrm{ab}}$ & $0.01^{\mathrm{a}}$ & $0.02^{\mathrm{a}}$ & $0.03^{\mathrm{a}}$ & $0.08^{\mathrm{a}}$ & $0.01^{\mathrm{a}}$ & $0.01^{\mathrm{a}}$ & $0.04^{\mathrm{a}}$ \\
\hline \multirow[t]{2}{*}{3} & $6.13 \pm$ & $6.21 \pm$ & $6.24 \pm$ & $6.26 \pm$ & $0.92 \pm$ & $0.87 \pm$ & $0.86 \pm$ & $0.84 \pm$ \\
\hline & $0.00^{\mathrm{b}}$ & $0.02^{\mathrm{ab}}$ & $0.03^{\mathrm{a}}$ & $0.02^{\mathrm{a}}$ & $0.02^{\mathrm{a}}$ & $0.04^{\mathrm{b}}$ & $0.08^{\mathrm{c}}$ & $0.00^{c}$ \\
\hline \multirow[t]{2}{*}{6} & $6.09 \pm$ & $6.12 \pm$ & $6.18 \pm$ & $6.21 \pm$ & $1.12 \pm$ & $1.02 \pm$ & $1.00 \pm$ & $0.96 \pm$ \\
\hline & $0.03^{\mathrm{b}}$ & $0.03^{\mathrm{ab}}$ & $0.04^{\mathrm{ab}}$ & $0.00^{\mathrm{a}}$ & $0.03^{\mathrm{a}}$ & $0.04^{\mathrm{b}}$ & $0.05^{\mathrm{b}}$ & $0.15^{\mathrm{bc}}$ \\
\hline \multirow[t]{2}{*}{9} & $5.98 \pm$ & $6.09 \pm$ & $6.12 \pm$ & $6.18 \pm$ & $1.21 \pm$ & $1.10 \pm$ & $1.07 \pm$ & $1.02 \pm$ \\
\hline & $0.02^{\mathrm{c}}$ & $0.03^{\mathrm{bc}}$ & $0.02^{\mathrm{a}}$ & $0.02^{\mathrm{a}}$ & $0.01^{\mathrm{a}}$ & $0.09^{\mathrm{b}}$ & $0.03^{\mathrm{b}}$ & $0.11^{\mathrm{c}}$ \\
\hline \multirow[t]{2}{*}{12} & $5.90 \pm$ & $5.97 \pm$ & $6.01 \pm$ & $6.11 \pm$ & $1.31 \pm$ & $1.18 \pm$ & $1.15 \pm$ & $1.08 \pm$ \\
\hline & $0.01^{\mathrm{c}}$ & $0.02^{\mathrm{bc}}$ & $0.00^{\mathrm{b}}$ & $0.03^{\mathrm{a}}$ & $0.07^{\mathrm{a}}$ & $0.01^{\mathrm{b}}$ & $0.08^{\mathrm{bc}}$ & $0.05^{\mathrm{c}}$ \\
\hline \multirow[t]{2}{*}{15} & $5.92 \pm$ & $5.95 \pm$ & $5.96 \pm$ & $5.99 \pm$ & $1.40 \pm$ & $1.26 \pm$ & $1.21 \pm$ & $1.13 \pm$ \\
\hline & $0.04^{\mathrm{b}}$ & $0.01^{\mathrm{ab}}$ & $0.02^{\mathrm{a}}$ & $0.05^{\mathrm{a}}$ & $0.01^{\mathrm{a}}$ & $0.08^{\mathrm{b}}$ & $0.01^{\mathrm{c}}$ & $0.06^{\mathrm{d}}$ \\
\hline \multirow[t]{2}{*}{18} & $5.93 \pm$ & $5.90 \pm$ & $5.92 \pm$ & $5.95 \pm$ & $1.49 \pm$ & $1.34 \pm$ & $1.28 \pm$ & $1.21 \pm$ \\
\hline & $0.02^{\mathrm{ab}}$ & $0.00^{\mathrm{ab}}$ & $0.01^{\mathrm{ab}}$ & $0.02^{\mathrm{a}}$ & $0.10^{\mathrm{a}}$ & $0.02^{\mathrm{b}}$ & $0.09^{c}$ & $0.01^{\mathrm{cd}}$ \\
\hline \multirow[t]{2}{*}{21} & $5.98 \pm$ & $6.08 \pm$ & $6.04 \pm$ & $6.05 \pm$ & $1.57 \pm$ & $1.39 \pm$ & $1.34 \pm$ & $1.27 \pm$ \\
\hline & $0.03^{\mathrm{b}}$ & $0.04^{\mathrm{b}}$ & $0.02^{\mathrm{ab}}$ & $0.01^{\mathrm{ab}}$ & $0.20^{\mathrm{a}}$ & $0.01^{\mathrm{b}}$ & $0.11^{\mathrm{c}}$ & $0.10^{\mathrm{d}}$ \\
\hline \multirow[t]{2}{*}{24} & $6.19 \pm$ & $6.17 \pm$ & $6.12 \pm$ & $6.09 \pm$ & $1.68 \pm$ & $1.47 \pm$ & $1.43 \pm$ & $1.34 \pm$ \\
\hline & $0.04^{\mathrm{ab}}$ & $0.02^{\mathrm{ab}}$ & $0.00^{\mathrm{a}}$ & $0.01^{\mathrm{a}}$ & $0.08^{\mathrm{a}}$ & $0.13^{\mathrm{bc}}$ & $0.12^{\mathrm{c}}$ & $0.09^{\mathrm{d}}$ \\
\hline \multirow[t]{2}{*}{27} & $6.34 \pm$ & $6.28 \pm$ & $6.24 \pm$ & $6.15 \pm$ & $1.77 \pm$ & $1.56 \pm$ & $1.50 \pm$ & $1.41 \pm$ \\
\hline & $0.02^{c}$ & $0.01^{\mathrm{ab}}$ & $0.03^{\mathrm{b}}$ & $0.02^{\mathrm{a}}$ & $0.01^{\mathrm{a}}$ & $0.19^{b}$ & $0.05^{\mathrm{c}}$ & $0.11^{\mathrm{d}}$ \\
\hline \multirow[t]{2}{*}{30} & $6.57 \pm$ & $6.39 \pm$ & $6.34 \pm$ & $6.21 \pm$ & $1.87 \pm$ & $1.69 \pm$ & $1.58 \pm$ & $1.50 \pm$ \\
\hline & $0.05^{\mathrm{c}}$ & $0.02^{\mathrm{bc}}$ & $0.01^{\mathrm{b}}$ & $0.03^{\mathrm{a}}$ & $0.12^{\mathrm{a}}$ & $0.18^{\mathrm{b}}$ & $0.17^{\mathrm{c}}$ & $0.13^{\mathrm{d}}$ \\
\hline
\end{tabular}

${ }^{\mathrm{a}-\mathrm{d}}$ Means within a raw with different superscript are significantly different $(\mathrm{P}<0.05)$.

Values are expressed as Mean \pm SD. 
nitrogen and total volatile basic nitrogen which increased the $\mathrm{pH}$ value of smoked samples. These results are in harmony with those reported by Sulieman (1993) and Gouda (2002).

\section{Thiobarbituric acid value (TBA)}

Table 4 shows the effect of cold storage on TBA value of smoked catfish fillets treated with phosphate. Values of TBA content significantly increased $(\mathrm{p}<0.05)$ during cold storage for all treatments. These increases might be due to some oxidation of lipids. Although, the TBA values of smoked fish fillets were increased during storage, it did not reach to the rejected level in all smoked samples due to the antioxidants of the smoke phenolic compounds (Said El-Din et al., 1996). Also, this probably may be due to the increased conversion of some unsaturated fatty acids on the surface into peroxides and some aldehydes and phenols in smoke might have become deposited on the fish which may be prevented the oxidative reactions of the fish oil (Saber et al., 1992). Data in the same Table show that, at the end of storage the highest TBA value was recorded in control sample followed by samples treated with 1 and $3 \%$ while the lowest value was observed in sample treated with 5\% STPP. The TBA values for control and sample treated with 1, 3 and 5\% STPP were $0.75,0.73,0.73$ and $0.71 \mathrm{mg}$ malonaldehyde $/ \mathrm{Kg}$ sample at zero time then, increased to $1.87,1.69,1.58$ and 1.50 at the end of the storage, respectively. The obtained results are in accordance with those of Abd El-Halim (2003), Goulas and Kontominos (2005) and Bilgin et al. (2008).

\section{Total volatile bases nitrogen (TVBN)}

The content of TVBN is useful for estimating the freshness of fish, and suggested 30 and 60 mg N/100 g. (on fresh weight basis) as the upper limit for fresh-water and marine fish; respectively. These results are in coincidence with those found by Bilgin et al. (2008) and Nahid et al. (2014).

Table 5 illustrates the effect of cold storage on TVBN of smoked catfish fillets treated with phosphate. Changes in TVBN content of the cold-smoked samples were significantly increased $(\mathrm{P}<0.05)$ during cold storage. TVBN contents were increased slowly during cold storage, especially before 18 days; particularly in samples treated with phosphate. The control, 1, 3 and 5\% STPP-treated samples contained 14.35, 14.32, 14.30 and $14.28 \mathrm{mg}$ TVBN/100 g. before storage, then increased to $34.45,28.38$, 24.65 and $21.11 \mathrm{mg}$ TVBN/100 g. after 30 days of cold storage, respectively.

\section{Trimethylamine nitrogen (TMAN)}

The maximum acceptable level of TMAN in smoked fish considered as $8.0-10.0 \mathrm{mg} / 100 \mathrm{~g}$ (Gouda, 2002). TMAN increased slightly within the first period of cold storage and rapidly up to the end of the storage for 30 days as given in Table 5.

The levels of TMAN contents significantly increased $(p<0.05)$ during cold storage for all treatments. Control samples recorded the highest increase. Also, it could be mentioned that, the treated fillets of smoked catfish contained less TMA than the untreated one at any given time of storage, especially with the increase of phosphate concentrations added. The smoked fillets contained 4.10, 3.85 and $3.52 \mathrm{mg}$ N/100 g. sample on fresh weight basis for the samples treated with 1, 3 and 5\% STPP after 30 days of storage, respectively in return $4.84 \mathrm{mg} / 100 \mathrm{~g}$. for the untreated sample. The decreases of mentioned nitrogenous compounds; particularly in case of treated-phosphate fillets may be due to phosphates used as antimicrobial agent to extend shelf life of fish. The obtained data are in accordance with those obtained by Goulas and Kontominos (2005) and Nahid et al. (2014).

\section{Effect of Cold Storage on Microbiological Quality of Cold-Smoked Catfish Fillets Treated With Different Concentrations of Phosphate}

\section{Total viable bacterial (TVBC) and E. coli counts}

Results obtained in Table 6 show the effect of cold storage on TVBC and E. coli counts of cold-smoked catfish fillets treated with different concentrations of phosphate. It was found that the levels of them were significantly increased $(p<0.05)$ during storage for all treatments. The initial counts of them in control fillets were higher than samples treated with phosphate. The 
Table 5. Changes in trimethylamine nitrogen (TMAN) and total volatile bases nitrogen (TVBN) $(\mathrm{mg} / 100 \mathrm{~g})$ contents of cold-smoked catfish fillets treated with 1,3 and $5 \%$ sodium tripolyphosphate (STPP) during cold storage at $4 \pm 1^{\circ} \mathrm{C}$ for 30 days (on fresh weight bases)

\begin{tabular}{|c|c|c|c|c|c|c|c|c|}
\hline \multirow{3}{*}{$\begin{array}{l}\text { Parameter } \\
\text { Storage } \\
\text { period (day) }\end{array}$} & \multicolumn{4}{|c|}{ TVBN (mg N/100 g) } & \multicolumn{4}{|c|}{ TMAN (mg N/100 g) } \\
\hline & \multicolumn{8}{|c|}{ STPP treatments } \\
\hline & Control & 1\% STPP & 3\% STPP & $5 \%$ STPP & Control & $1 \%$ STPP & 3\% STPP & $5 \%$ STPP \\
\hline \multirow[t]{2}{*}{$\mathbf{0}$} & $14.35 \pm$ & $14.32 \pm$ & $14.30 \pm$ & $14.28 \pm$ & $1.02 \pm$ & $1.02 \pm$ & $1.02 \pm$ & $1.02 \pm$ \\
\hline & $0.96^{\mathrm{a}}$ & $1.02^{\mathrm{a}}$ & $1.07^{\mathrm{a}}$ & $0.76^{\mathrm{a}}$ & $0.10^{\mathrm{a}}$ & $0.12^{\mathrm{a}}$ & $0.11^{\mathrm{a}}$ & $0.12^{\mathrm{a}}$ \\
\hline \multirow[t]{2}{*}{3} & $15.24 \pm$ & $15.15 \pm$ & $15.08 \pm$ & $14.73 \pm$ & $1.35 \pm$ & $1.30 \pm$ & $1.25 \pm$ & $1.24 \pm$ \\
\hline & $0.86^{\mathrm{a}}$ & $0.89^{\mathrm{a}}$ & $1.01^{\mathrm{a}}$ & $1.02^{\mathrm{a}}$ & $0.12^{\mathrm{a}}$ & $0.11^{\mathrm{a}}$ & $0.16^{\mathrm{b}}$ & $0.15^{\mathrm{b}}$ \\
\hline \multirow[t]{2}{*}{6} & $16.61 \pm$ & $15.83 \pm$ & $15.45 \pm$ & $15.15 \pm$ & $1.70 \pm$ & $1.61 \pm$ & $1.51 \pm$ & $1.46 \pm$ \\
\hline & $1.01^{\mathrm{a}}$ & $1.16^{\mathrm{a}}$ & $1.21^{\mathrm{ab}}$ & $1.01^{\mathrm{b}}$ & $0.11^{\mathrm{a}}$ & $0.14^{\mathrm{ab}}$ & $0.19^{\mathrm{b}}$ & $0.19^{\mathrm{b}}$ \\
\hline \multirow[t]{2}{*}{9} & $17.78 \pm$ & $17.14 \pm$ & $16.61 \pm$ & $15.93 \pm$ & $2.02 \pm$ & $1.89 \pm$ & $1.77 \pm$ & $1.70 \pm$ \\
\hline & $1.42^{\mathrm{a}}$ & $1.00^{\mathrm{ab}}$ & $0.78^{\mathrm{b}}$ & $1.32^{\mathrm{c}}$ & $0.21^{\mathrm{a}}$ & $0.18^{\mathrm{ab}}$ & $0.21^{\mathrm{b}}$ & $0.15^{\mathrm{b}}$ \\
\hline \multirow[t]{2}{*}{12} & $18.85 \pm$ & $18.21 \pm$ & $17.19 \pm$ & $16.13 \pm$ & $2.39 \pm$ & $2.24 \pm$ & $2.06 \pm$ & $1.94 \pm$ \\
\hline & $1.30^{\mathrm{a}}$ & $0.86^{\mathrm{ab}}$ & $0.96^{\mathrm{bc}}$ & $1.20^{\mathrm{c}}$ & $0.18^{\mathrm{a}}$ & $0.26^{\mathrm{ab}}$ & $0.25^{\mathrm{bc}}$ & $0.21^{\mathrm{c}}$ \\
\hline \multirow[t]{2}{*}{15} & $20.38 \pm$ & $19.10 \pm$ & $18.23 \pm$ & $17.14 \pm$ & $2.75 \pm$ & $2.52 \pm$ & $2.35 \pm$ & $2.16 \pm$ \\
\hline & $1.73^{\mathrm{a}}$ & $1.51^{\mathrm{b}}$ & $1.01^{\mathrm{bc}}$ & $1.11^{\mathrm{c}}$ & $0.10^{\mathrm{a}}$ & $0.20^{\mathrm{b}}$ & $0.31^{\mathrm{b}}$ & $0.32^{\mathrm{c}}$ \\
\hline \multirow[t]{2}{*}{18} & $23.29 \pm$ & $21.48 \pm$ & $19.35 \pm$ & $17.84 \pm$ & $3.14 \pm$ & $2.84 \pm$ & $2.64 \pm$ & $2.39 \pm$ \\
\hline & $1.78^{\mathrm{a}}$ & $1.40^{\mathrm{b}}$ & $1.23^{\mathrm{bc}}$ & $1.00^{\mathrm{c}}$ & $0.26^{\mathrm{a}}$ & $0.25^{\mathrm{b}}$ & $0.23^{\mathrm{b}}$ & $0.34^{\mathrm{c}}$ \\
\hline \multirow[t]{2}{*}{21} & $26.27 \pm$ & $23.98 \pm$ & $21.42 \pm$ & $18.56 \pm$ & $3.52 \pm$ & $3.18 \pm$ & $2.90 \pm$ & $2.62 \pm$ \\
\hline & $1.30^{\mathrm{a}}$ & $1.46^{\mathrm{b}}$ & $1.34^{\mathrm{bc}}$ & $1.21^{\mathrm{cd}}$ & $0.43^{\mathrm{a}}$ & $0.32^{\mathrm{bc}}$ & $0.36^{\mathrm{bc}}$ & $0.28^{\mathrm{c}}$ \\
\hline \multirow[t]{2}{*}{24} & $29.18 \pm$ & $25.38 \pm$ & $22.46 \pm$ & $19.23 \pm$ & $3.93 \pm$ & $3.49 \pm$ & $3.16 \pm$ & $2.88 \pm$ \\
\hline & $1.96^{\mathrm{a}}$ & $1.08^{\mathrm{b}}$ & $1.56^{\mathrm{C}}$ & $1.32^{\mathrm{cd}}$ & $0.23^{\mathrm{a}}$ & $0.16^{\mathrm{b}}$ & $0.41^{\mathrm{bc}}$ & $0.27^{\mathrm{cd}}$ \\
\hline \multirow[t]{2}{*}{27} & $31.81 \pm$ & $26.85 \pm$ & $23.52 \pm$ & $20.00 \pm$ & $4.35 \pm$ & $3.85 \pm$ & $3.48 \pm$ & $3.23 \pm$ \\
\hline & $1.46^{\mathrm{a}}$ & $1.86^{\mathrm{b}}$ & $1.72^{\mathrm{c}}$ & $1.41^{\mathrm{d}}$ & $0.42^{\mathrm{a}}$ & $0.37^{\mathrm{b}}$ & $0.43^{c}$ & $0.31^{\mathrm{cd}}$ \\
\hline \multirow[t]{2}{*}{30} & $34.45 \pm$ & $28.38 \pm$ & $24.65 \pm$ & $21.11 \pm$ & $4.84 \pm$ & $4.10 \pm$ & $3.85 \pm$ & $3.52 \pm$ \\
\hline & $1.52^{\mathrm{a}}$ & $1.20^{\mathrm{b}}$ & $1.90^{c}$ & $1.46^{\mathrm{d}}$ & $0.51^{\mathrm{a}}$ & $0.34^{\mathrm{b}}$ & $0.30^{\text {cd }}$ & $0.41^{\mathrm{d}}$ \\
\hline
\end{tabular}

${ }^{\mathrm{a}-\mathrm{d}}$ Means within a raw with different superscript are significantly different $(\mathrm{P}<0.05)$.

Values are expressed as Mean \pm SD. 
Table 6. Changes in total viable bacterial (TVBC) and $E$. coli counts $\left(\log _{10}\right.$ CFU/g.) of coldsmoked catfish fillets treated with 1, 3 and $5 \%$ sodium tripolyphosphate (STPP) during cold storage at $4 \pm 1^{\circ} \mathrm{C}$ for 30 days

\begin{tabular}{|c|c|c|c|c|c|c|c|c|}
\hline \multirow{3}{*}{$\begin{array}{l}\text { Parameter } \\
\text { Storage } \\
\text { period } \\
\text { (day) }\end{array}$} & \multicolumn{4}{|c|}{ TVBC ( $\log _{10}$ CFU/g.) } & \multicolumn{4}{|c|}{ E. coli $\left(\log _{10} \mathrm{CFU} / \mathrm{g}.\right)$} \\
\hline & \multicolumn{8}{|c|}{ STPP treatments } \\
\hline & Control & $1 \%$ STPP & $3 \%$ STPP & $5 \%$ STPP & Control & $1 \%$ STPP & 3\% STPP & 5\% STPP \\
\hline \multirow[t]{2}{*}{$\mathbf{0}$} & $2.65 \pm$ & $2.23 \pm$ & $2.18 \pm$ & $2.00 \pm$ & $0.88 \pm$ & $0.70 \pm$ & $0.64 \pm$ & $0.53 \pm$ \\
\hline & $0.15^{\mathrm{a}}$ & $0.13^{\mathrm{a}}$ & $0.11^{\mathrm{a}}$ & $0.14^{\mathrm{a}}$ & $0.05^{\mathrm{a}}$ & $0.09^{\mathrm{a}}$ & $0.11^{\mathrm{ab}}$ & $0.01^{\mathrm{b}}$ \\
\hline \multirow[t]{2}{*}{3} & $3.00 \pm$ & $2.35 \pm$ & $2.29 \pm$ & $2.15 \pm$ & $1.06 \pm$ & $0.93 \pm$ & $0.71 \pm$ & $0.60 \pm$ \\
\hline & $0.19^{\mathrm{a}}$ & $0.18^{\mathrm{ab}}$ & $0.15^{\mathrm{ab}}$ & $0.14^{\mathrm{b}}$ & $0.04^{\mathrm{a}}$ & $0.11^{\mathrm{a}}$ & $0.12^{\mathrm{ab}}$ & $0.06^{\mathrm{b}}$ \\
\hline \multirow[t]{2}{*}{6} & $3.13 \pm$ & $2.44 \pm$ & $2.38 \pm$ & $2.32 \pm$ & $1.10 \pm$ & $1.06 \pm$ & $1.09 \pm$ & $0.87 \pm$ \\
\hline & $0.13^{\mathrm{a}}$ & $0.17^{\mathrm{b}}$ & $0.12^{\mathrm{b}}$ & $0.19^{\mathrm{b}}$ & $0.08^{\mathrm{a}}$ & $0.14^{\mathrm{ab}}$ & $0.10^{\mathrm{b}}$ & $0.08^{\mathrm{b}}$ \\
\hline \multirow[t]{2}{*}{9} & $3.21 \pm$ & $2.56 \pm$ & $2.42 \pm$ & $2.38 \pm$ & $1.68 \pm$ & $1.26 \pm$ & $1.14 \pm$ & $0.92 \pm$ \\
\hline & $0.14^{\mathrm{a}}$ & $0.19^{b}$ & $0.11^{\mathrm{bc}}$ & $0.16^{\mathrm{c}}$ & $0.11^{\mathrm{a}}$ & $0.16^{\mathrm{b}}$ & $0.14^{\mathrm{b}}$ & $0.11_{c}$ \\
\hline \multirow[t]{2}{*}{12} & $3.32 \pm$ & $2.75 \pm$ & $2.49 \pm$ & $2.40 \pm$ & $2.11 \pm$ & $1.59 \pm$ & $1.32 \pm$ & $1.08 \pm$ \\
\hline & $0.15^{\mathrm{a}}$ & $0.15^{\mathrm{b}}$ & $0.16^{\mathrm{bc}}$ & $0.19^{\mathrm{bc}}$ & $0.12^{\mathrm{a}}$ & $0.10^{\mathrm{b}}$ & $0.11^{\mathrm{c}}$ & $0.13^{\mathrm{d}}$ \\
\hline \multirow[t]{2}{*}{15} & $3.45 \pm$ & $2.92 \pm$ & $2.56 \pm$ & $2.53 \pm$ & $2.42 \pm$ & $2.06 \pm$ & $1.85 \pm$ & $1.26 \pm$ \\
\hline & $0.11^{\mathrm{a}}$ & $0.17^{\mathrm{b}}$ & $0.17^{\mathrm{bc}}$ & $0.20^{c}$ & $0.10^{\mathrm{a}}$ & $0.19^{\mathrm{b}}$ & $0.20^{\mathrm{bc}}$ & $0.16^{\mathrm{d}}$ \\
\hline \multirow[t]{2}{*}{18} & $3.52 \pm$ & $3.13 \pm$ & $2.75 \pm$ & $2.60 \pm$ & $2.84 \pm$ & $2.32 \pm$ & $2.08 \pm$ & $1.54 \pm$ \\
\hline & $0.10^{\mathrm{a}}$ & $0.15^{\mathrm{b}}$ & $0.10^{\mathrm{c}}$ & $0.22^{\mathrm{c}}$ & $0.09^{\mathrm{a}}$ & $0.20^{\mathrm{b}}$ & $0.18^{\mathrm{bc}}$ & $0.19^{\mathrm{d}}$ \\
\hline \multirow[t]{2}{*}{21} & $3.63 \pm$ & $3.29 \pm$ & $2.90 \pm$ & $2.71 \pm$ & $3.15 \pm$ & $2.58 \pm$ & $2.29 \pm$ & $1.85 \pm$ \\
\hline & $0.15^{\mathrm{a}}$ & $0.19^{b}$ & $0.19^{c}$ & $0.14^{\mathrm{c}}$ & $0.13^{\mathrm{a}}$ & $0.22^{\mathrm{b}}$ & $0.19^{\mathrm{c}}$ & $0.16^{\mathrm{d}}$ \\
\hline \multirow[t]{2}{*}{24} & $3.81 \pm$ & $3.43 \pm$ & $3.16 \pm$ & $2.84 \pm$ & $3.57 \pm$ & $2.69 \pm$ & $2.56 \pm$ & $2.04 \pm$ \\
\hline & $0.18^{\mathrm{a}}$ & $0.22^{\mathrm{b}}$ & $0.20^{\mathrm{c}}$ & $0.11^{\mathrm{d}}$ & $0.19^{\mathrm{a}}$ & $0.21^{\mathrm{b}}$ & $0.21^{\mathrm{c}}$ & $0.22^{\mathrm{d}}$ \\
\hline \multirow[t]{2}{*}{27} & $4.18 \pm$ & $3.65 \pm$ & $3.30 \pm$ & $3.07 \pm$ & $3.81 \pm$ & $2.94 \pm$ & $2.73 \pm$ & $2.27 \pm$ \\
\hline & $0.19^{\mathrm{a}}$ & $0.19^{b}$ & $0.21^{\mathrm{cd}}$ & $0.15^{\mathrm{d}}$ & $0.18^{\mathrm{a}}$ & $0.18^{\mathrm{b}}$ & $0.22^{\mathrm{C}}$ & $0.20^{\mathrm{d}}$ \\
\hline \multirow[t]{2}{*}{30} & $4.86 \pm$ & $3.96 \pm$ & $3.53 \pm$ & $3.26 \pm$ & $4.26 \pm$ & $3.37 \pm$ & $3.08 \pm$ & $2.50 \pm$ \\
\hline & $0.22^{\mathrm{a}}$ & $0.21^{\mathrm{b}}$ & $0.25^{\mathrm{c}}$ & $0.19^{\mathrm{d}}$ & $0.29^{\mathrm{a}}$ & $0.23^{\mathrm{b}}$ & $0.18^{\mathrm{C}}$ & $0.17^{\mathrm{d}}$ \\
\hline
\end{tabular}

\footnotetext{
${ }^{\mathrm{a}-\mathrm{d}}$ Means within a raw with different superscript are significantly different $(\mathrm{P}<0.05)$.
}

Values are expressed as Mean \pm SD. 
total bacterial counts multiplication were slowly increased during 30 days of cold storage. The counts were 2.65, 2.23, 2.18 and $2.00 \log _{10}$ CFU/g, in control and samples treated with 1, 3 and 5\% STPP, respectively. Consequently, the progressive increases of bacteria were markedly till the end of the storage period. Although, 5\% STPP treated samples reflected the lowest count $3.26 \log _{10} \mathrm{CFU} / \mathrm{g}$, the untreated sample (control) reflected the highest count $4.86 \log _{10} \mathrm{CFU} / \mathrm{g}$. $E$. coli count was significantly increased $(\mathrm{p}<0.05)$ during storage period. It was found to be, 0.88 , $0.70,0.64$ and $0.53 \log _{10} \mathrm{CFU} / \mathrm{g}$, for coldsmoked fillets control, 1, 3 and 5\% STPP, respectively at zero time of storage. While, at the end of the storage period they were 4.26, 3.37, 3.08 and $2.50 \log _{10} \mathrm{CFU} / \mathrm{g}$., respectively. This may be attributed to handling during preparation of whole fish to fillets for smoking. On the other hand, phosphates have been used as a slight antimicrobial surface treatment agents to decrease populations of pathogens, prevent growth of spoilage microorganisms, and extend the shelf-life of fish (Kim and Marshall 1999). The obtained data are in accordance with those reported by El-Akeel (1988), Goulas and Kontominos (2005), Bilgin et al. (2008) and Egbal et al. (2013).

\section{Pyschrophilic bacterial count (PsBC)}

Results obtained in Table 7 show the effect of cold storage on pyschrophilic bacterial count of cold-smoked catfish fillets treated with different concentrations of phosphate. Results show that pyschrophilic bacterial count significantly increased $(\mathrm{p}<0.05)$ during storage period for all samples. Pyschrophilic bacterial count was detected at low count for all samples at zero time of storage period, while at the end of storage period were 3.92, 3.48, 3.31 and 2.82 $\log _{10} \mathrm{CFU} / \mathrm{g}$, for control, 1,3 and 5\% STPP treated samples, respectively. Data in the same Table show that, the lowest value of pyschrophilic bacterial count was recorded in samples treated with phosphate and the highest level was in control sample. However, the pyschrophilic bacteria gave high values throughout storage period which may be due to the presence of pyschrophilic spores forming bacteria which are again activated by cooling. This obtained data are in accordance with those stated by El-Akeel (1988), Goulas and Kontominos (2005) and Egbal et al. (2013).

\section{Mould and yeast counts (MYC)}

Data illustrated in Table 7 shows the effect of cold storage on moulds and yeast counts of coldsmoked catfish fillets treated with different concentrations of phosphate.

The counts of moulds and yeasts significantly increased $(\mathrm{p}<0.05)$ during storage period. It was not detected for all samples at zero time of the storage period, while the increase during storage was observed for all treatments till the end of storage period which were 4.05, 3.11, 2.88 and $2.62 \log _{10} \mathrm{CFU} / \mathrm{g}$, for control, 1, 3 and 5\% STPP treated samples, respectively. The lowest numbers of mould and yeast were recorded in samples treated with phosphate and the highest value was detected in control sample. These obtained data are in accordance with those mentioned by El-Akeel (1988), Goulas and Kontominos (2005), Yanar (2007) and Egbal et al. (2013).

\section{Effect of Cold Storage on Organoleptic Properties of Cold-Smoked Catfish Fillets Treated with Different Concentrations of Phosphate}

Data in Table 8 shows the effect of cold storage on colour and odour of cold-smoked catfish fillets treated with different concentrations of phosphate.

Sensory properties were significantly decreased $(\mathrm{p}<0.05)$ during storage period. Colour score was 8.50 for all samples at zero time of storage period, while at the end of storage period they were 4.60, 5.30, 5.85 and 6.50 for control, 1, 3 and 5\% STPP treated samples, respectively. Odour score was 9.20 for all samples, while at the end of storage period they were 5.07, 5.65, 6.30 and 6.80 for control, 1, 3 and 5\% STPP treated samples, respectively. Results also show that, the highest grades in colour and odour were found for samples treated with phosphate.

Additionally, data presented in Table 9 shows the effect of cold storage on taste and texture of cold-smoked catfish fillets treated with different concentrations of phosphate. 
Table 7. Changes in psychrophilic bacterial count as well as mold and yeast counts $\left(\log _{10}\right.$ CFU/g.) of cold-smoked catfish fillets treated with 1, 3 and 5\% sodium tripolyphosphate (STPP) during cold storage at $4 \pm 1^{\circ} \mathrm{C}$ for 30 days

\begin{tabular}{|c|c|c|c|c|c|c|c|c|}
\hline \multirow{3}{*}{$\begin{array}{l}\text { Parameter } \\
\text { Storage } \\
\text { period } \\
\text { (day) }\end{array}$} & \multicolumn{4}{|c|}{ Psychrophilic ( $\log _{10}$ CFU/g.) } & \multicolumn{4}{|c|}{ Mold and Yeast ( $\log _{10} \mathrm{CFU} / \mathrm{g}$.) } \\
\hline & \multicolumn{8}{|c|}{ STPP treatments } \\
\hline & Control & 1\% STPP & $3 \%$ STPP & $5 \%$ STPP & Control & $1 \%$ STPP & \% STPP & $\%$ STPP \\
\hline \multirow[t]{2}{*}{$\mathbf{0}$} & $0.69 \pm$ & $0.60 \pm$ & $0.45 \pm$ & $0.40 \pm$ & ND* & ND* & $\mathrm{ND}^{*}$ & ND* \\
\hline & $0.03^{\mathrm{a}}$ & $0.09^{\mathrm{a}}$ & $0.05^{\mathrm{a}}$ & $0.02^{\mathrm{a}}$ & & & & \\
\hline \multirow[t]{2}{*}{3} & $1.69 \pm$ & $1.41 \pm$ & $1.20 \pm$ & $0.72 \pm$ & $1.30 \pm$ & $1.11 \pm$ & ND* & ND* \\
\hline & $0.11^{\mathrm{a}}$ & $0.14^{\mathrm{a}}$ & $0.19^{\mathrm{a}}$ & $0.11^{\mathrm{ab}}$ & $0.14^{\mathrm{a}}$ & $0.11^{\mathrm{b}}$ & & \\
\hline \multirow[t]{2}{*}{6} & $1.85 \pm$ & $1.76 \pm$ & $1.57 \pm$ & $0.95 \pm$ & $1.55 \pm$ & $1.34 \pm$ & $1.17 \pm$ & $1.15 \pm$ \\
\hline & $0.12^{\mathrm{a}}$ & $0.11^{\mathrm{a}}$ & $0.15^{\mathrm{ab}}$ & $0.13^{\mathrm{b}}$ & $0.11^{\mathrm{a}}$ & $0.12^{\mathrm{ab}}$ & $0.10^{\mathrm{b}}$ & $0.07^{\mathrm{b}}$ \\
\hline \multirow[t]{2}{*}{9} & $1.88 \pm$ & $1.82 \pm$ & $1.74 \pm$ & $1.21 \pm$ & $1.73 \pm$ & $1.54 \pm$ & $1.35 \pm$ & $1.27 \pm$ \\
\hline & $0.10^{\mathrm{a}}$ & $0.12^{\mathrm{a}}$ & $0.12^{\mathrm{ab}}$ & $0.16^{\mathrm{b}}$ & $0.12^{\mathrm{a}}$ & $0.12^{\mathrm{ab}}$ & $0.12^{\mathrm{b}}$ & $0.11^{\mathrm{b}}$ \\
\hline \multirow[t]{2}{*}{12} & $2.27 \pm$ & $2.06 \pm$ & $1.90 \pm$ & $1.40 \pm$ & $2.14 \pm$ & $1.87 \pm$ & $1.67 \pm$ & $1.54 \pm$ \\
\hline & $0.13^{\mathrm{a}}$ & $0.13^{\mathrm{ab}}$ & $0.11^{\mathrm{ab}}$ & $0.13^{\mathrm{b}}$ & $0.13^{\mathrm{a}}$ & $0.10^{\mathrm{ab}}$ & $0.10^{\mathrm{ab}}$ & $0.15^{\mathrm{b}}$ \\
\hline \multirow[t]{2}{*}{15} & $2.43 \pm$ & $2.26 \pm$ & $2.12 \pm$ & $1.75 \pm$ & $2.43 \pm$ & $2.21 \pm$ & $2.00 \pm$ & $1.76 \pm$ \\
\hline & $0.19^{\mathrm{a}}$ & $0.11^{\mathrm{b}}$ & $0.10^{\mathrm{b}}$ & $0.11^{\mathrm{bc}}$ & $0.13^{\mathrm{a}}$ & $0.15^{\mathrm{b}}$ & $0.21^{\mathrm{b}}$ & $0.14^{\mathrm{c}}$ \\
\hline \multirow[t]{2}{*}{18} & $2.62 \pm$ & $2.37 \pm$ & $2.28 \pm$ & $1.90 \pm$ & $2.67 \pm$ & $2.37 \pm$ & $2.24 \pm$ & $1.95 \pm$ \\
\hline & $0.18^{\mathrm{a}}$ & $0.13^{\mathrm{b}}$ & $0.15^{\mathrm{b}}$ & $0.20^{c}$ & $0.11^{\mathrm{a}}$ & $0.12^{\mathrm{b}}$ & $0.19^{\mathrm{b}}$ & $0.13^{c}$ \\
\hline \multirow[t]{2}{*}{21} & $2.84 \pm$ & $2.71 \pm$ & $2.67 \pm$ & $2.21 \pm$ & $2.98 \pm$ & $2.45 \pm$ & $2.31 \pm$ & $1.97 \pm$ \\
\hline & $0.20^{\mathrm{a}}$ & $0.15^{\mathrm{ab}}$ & $0.18^{\mathrm{b}}$ & $0.19^{\mathrm{c}}$ & $0.20^{\mathrm{a}}$ & $0.20^{\mathrm{b}}$ & $0.14^{\mathrm{bc}}$ & $0.11^{\mathrm{c}}$ \\
\hline \multirow[t]{2}{*}{24} & $3.26 \pm$ & $2.91 \pm$ & $2.86 \pm$ & $2.43 \pm$ & $3.23 \pm$ & $2.72 \pm$ & $2.48 \pm$ & $2.22 \pm$ \\
\hline & $0.27^{\mathrm{a}}$ & $0.18^{\mathrm{b}}$ & $0.15^{\mathrm{b}}$ & $0.17^{\mathrm{c}}$ & $0.15^{\mathrm{a}}$ & $0.18^{\mathrm{b}}$ & $0.16^{c}$ & $0.14^{\mathrm{d}}$ \\
\hline \multirow[t]{2}{*}{27} & $3.55 \pm$ & $3.22 \pm$ & $3.11 \pm$ & $2.69 \pm$ & $3.62 \pm$ & $2.85 \pm$ & $2.63 \pm$ & $2.35 \pm$ \\
\hline & $0.31^{\mathrm{a}}$ & $0.19^{\mathrm{b}}$ & $0.21^{\mathrm{bc}}$ & $0.20^{\mathrm{d}}$ & $0.22^{\mathrm{a}}$ & $0.21^{\mathrm{b}}$ & $0.21^{\mathrm{bc}}$ & $0.22^{\mathrm{d}}$ \\
\hline \multirow[t]{2}{*}{30} & $3.92 \pm$ & $3.48 \pm$ & $3.31 \pm$ & $2.82 \pm$ & $4.05 \pm$ & $3.11 \pm$ & $2.88 \pm$ & $2.62 \pm$ \\
\hline & $0.26^{\mathrm{a}}$ & $0.21^{\mathrm{b}}$ & $0.19^{c}$ & $0.22^{\mathrm{d}}$ & $0.23^{\mathrm{a}}$ & $0.19^{\mathrm{b}}$ & $0.17^{\mathrm{c}}$ & $0.14^{d}$ \\
\hline
\end{tabular}

${ }^{\mathrm{a}-\mathrm{d}}$ Means within a raw with different superscript are significantly different $(\mathrm{P}<0.05)$.

Values are expressed as Mean \pm SD. $\quad *$ ND: Not detected. 
Table 8. Changes in colour and odour of cold-smoked catfish fillets treated with 1,3 and $5 \%$ sodium tripolyphosphate (STPP) during cold storage at $4 \pm 1^{\circ} \mathrm{C}$ for 30 days

\begin{tabular}{|c|c|c|c|c|c|c|c|c|}
\hline \multirow{3}{*}{$\begin{array}{l}\text { Parameter } \\
\text { Storage } \\
\text { period } \\
\text { (day) }\end{array}$} & \multicolumn{4}{|c|}{ Colour } & \multicolumn{4}{|c|}{ Odour } \\
\hline & \multicolumn{8}{|c|}{ STPP treatments } \\
\hline & Control & 1\% STPP & $3 \%$ STPP & $5 \%$ STPP & Control & 1\% STPP & 3\% STPP & $5 \%$ STPP \\
\hline \multirow[t]{2}{*}{0} & $8.50 \pm$ & $8.50 \pm$ & $8.50 \pm$ & $8.50 \pm$ & $9.20 \pm$ & $9.20 \pm$ & $9.20 \pm$ & $9.20 \pm$ \\
\hline & $0.21^{\mathrm{a}}$ & $0.19^{\mathrm{a}}$ & $0.18^{\mathrm{a}}$ & $0.17^{\mathrm{a}}$ & $0.19^{\mathrm{a}}$ & $0.20^{\mathrm{a}}$ & $0.22^{\mathrm{a}}$ & $0.17^{\mathrm{a}}$ \\
\hline \multirow[t]{2}{*}{3} & $8.10 \pm$ & $8.15 \pm$ & $8.34 \pm$ & $8.45 \pm$ & $8.95 \pm$ & $9.00 \pm$ & $9.10 \pm$ & $9.15 \pm$ \\
\hline & $0.15^{\mathrm{ab}}$ & $0.15^{\mathrm{ab}}$ & $0.19^{\mathrm{a}}$ & $0.18^{\mathrm{a}}$ & $0.20^{\mathrm{a}}$ & $0.19^{\mathrm{a}}$ & $0.19^{\mathrm{a}}$ & $0.19^{\mathrm{a}}$ \\
\hline \multirow[t]{2}{*}{6} & $7.80 \pm$ & $7.95 \pm$ & $8.11 \pm$ & $8.30 \pm$ & $8.50 \pm$ & $8.63 \pm$ & $8.74 \pm$ & $8.88 \pm$ \\
\hline & $0.19^{\mathrm{b}}$ & $0.11^{\mathrm{ab}}$ & $0.20^{\mathrm{ab}}$ & $0.19^{\mathrm{a}}$ & $0.18^{\mathrm{ab}}$ & $0.18^{\mathrm{a}}$ & $0.16^{\mathrm{a}}$ & $0.15^{\mathrm{a}}$ \\
\hline \multirow[t]{2}{*}{9} & $7.42 \pm$ & $7.67 \pm$ & $7.85 \pm$ & $8.17 \pm$ & $8.15 \pm$ & $8.31 \pm$ & $8.45 \pm$ & $8.70 \pm$ \\
\hline & $0.18^{c}$ & $0.14^{\mathrm{b}}$ & $0.12^{\mathrm{b}}$ & $0.16^{\mathrm{a}}$ & $0.16^{\mathrm{b}}$ & $0.1^{9 \mathrm{ab}}$ & $0.20^{\mathrm{ab}}$ & $0.19^{\mathrm{a}}$ \\
\hline \multirow[t]{2}{*}{12} & $7.03 \pm$ & $7.35 \pm$ & $7.65 \pm$ & $7.94 \pm$ & $7.64 \pm$ & $7.98 \pm$ & $8.10 \pm$ & $8.55 \pm$ \\
\hline & $0.21^{\mathrm{c}}$ & $0.13^{\mathrm{b}}$ & $0.15^{\mathrm{b}}$ & $0.19^{\mathrm{a}}$ & $0.19^{\mathrm{b}}$ & $0.19^{\mathrm{ab}}$ & $0.20^{\mathrm{ab}}$ & $0.14^{\mathrm{a}}$ \\
\hline \multirow[t]{2}{*}{15} & $6.60 \pm$ & $7.00 \pm$ & $7.40 \pm$ & $7.75 \pm$ & $7.23 \pm$ & $7.40 \pm$ & $7.80 \pm$ & $8.40 \pm$ \\
\hline & $0.13^{\mathrm{c}}$ & $0.16^{\mathrm{bc}}$ & $0.12^{\mathrm{b}}$ & $0.12^{\mathrm{a}}$ & $0.17^{\mathrm{bc}}$ & $0.15^{\mathrm{b}}$ & $0.19^{\mathrm{b}}$ & $0.16^{\mathrm{a}}$ \\
\hline \multirow[t]{2}{*}{18} & $6.25 \pm$ & $6.65 \pm$ & $7.16 \pm$ & $7.50 \pm$ & $6.80 \pm$ & $7.15 \pm$ & $7.55 \pm$ & $8.00 \pm$ \\
\hline & $0.15^{\mathrm{d}}$ & $0.12^{\mathrm{c}}$ & $0.16^{\mathrm{b}}$ & $0.10^{\mathrm{a}}$ & $0.17^{\mathrm{c}}$ & $0.18^{\mathrm{b}}$ & $0.15^{\mathrm{b}}$ & $0.17^{\mathrm{a}}$ \\
\hline \multirow[t]{2}{*}{21} & $5.70 \pm$ & $6.35 \pm$ & $6.61 \pm$ & $7.26 \pm$ & $6.45 \pm$ & $6.50 \pm$ & $7.20 \pm$ & $7.81 \pm$ \\
\hline & $0.11^{\mathrm{c}}$ & $0.15^{\mathrm{b}}$ & $0.18^{\mathrm{b}}$ & $0.15^{\mathrm{a}}$ & $0.11^{\mathrm{c}}$ & $0.16^{\mathrm{bc}}$ & $0.14^{\mathrm{b}}$ & $0.15^{\mathrm{a}}$ \\
\hline \multirow[t]{2}{*}{24} & $5.20 \pm$ & $6.00 \pm$ & $6.30 \pm$ & $6.90 \pm$ & $5.75 \pm$ & $6.24 \pm$ & $6.90 \pm$ & $7.58 \pm$ \\
\hline & $0.10^{\mathrm{cd}}$ & $0.11^{\mathrm{c}}$ & $0.15^{\mathrm{b}}$ & $0.19^{\mathrm{a}}$ & $0.12^{d}$ & $0.16^{\mathrm{c}}$ & $0.14^{\mathrm{b}}$ & $0.14^{\mathrm{a}}$ \\
\hline \multirow[t]{2}{*}{27} & $4.75 \pm$ & $5.80 \pm$ & $6.04 \pm$ & $6.77 \pm$ & $5.40 \pm$ & $5.80 \pm$ & $6.60 \pm$ & $7.25 \pm$ \\
\hline & $0.17^{\mathrm{d}}$ & $0.12^{\mathrm{c}}$ & $0.12^{\mathrm{b}}$ & $0.13^{\mathrm{a}}$ & $0.13^{\mathrm{d}}$ & $0.13^{\mathrm{c}}$ & $0.12^{\mathrm{b}}$ & $0.13^{\mathrm{a}}$ \\
\hline \multirow[t]{2}{*}{30} & $4.60 \pm$ & $5.30 \pm$ & $5.85 \pm$ & $6.50 \pm$ & $5.07 \pm$ & $5.65 \pm$ & $6.30 \pm$ & $6.80 \pm$ \\
\hline & $0.11^{\mathrm{d}}$ & $0.10^{c}$ & $0.16^{\mathrm{b}}$ & $0.10^{\mathrm{a}}$ & $0.17^{\mathrm{d}}$ & $0.11^{\mathrm{c}}$ & $0.14^{\mathrm{b}}$ & $0.10^{\mathrm{a}}$ \\
\hline
\end{tabular}

${ }^{\mathrm{a}-\mathrm{d}}$ Means within a raw with different superscript are significantly different $(\mathrm{P}<0.05)$.

Values are expressed as Mean \pm SD. 
Table 9. Changes in taste and texture of cold-smoked catfish fillets treated with 1,3 and $5 \%$ sodium tripolyphosphate (STPP) during cold storage at $4 \pm 1^{\circ} \mathrm{C}$ for 30 days

\begin{tabular}{|c|c|c|c|c|c|c|c|c|}
\hline \multirow{3}{*}{$\begin{array}{l}\text { Parameter } \\
\text { Storage } \\
\text { period } \\
\text { (day) }\end{array}$} & \multicolumn{4}{|c|}{ Taste } & \multicolumn{4}{|c|}{ Texture } \\
\hline & \multicolumn{8}{|c|}{ STPP treatments } \\
\hline & Control & $1 \%$ STPP & 3\% STPP & $5 \%$ STPP & Control & $1 \%$ STPP & 3\% STPP & $5 \%$ STPP \\
\hline \multirow[t]{2}{*}{$\mathbf{0}$} & $9.00 \pm$ & $9.00 \pm$ & $9.00 \pm$ & $9.00 \pm$ & $9.50 \pm$ & $9.50 \pm$ & $9.50 \pm$ & $9.50 \pm$ \\
\hline & $0.22^{\mathrm{a}}$ & $0.20^{\mathrm{a}}$ & $0.19^{\mathrm{a}}$ & $0.20^{\mathrm{a}}$ & $0.32^{\mathrm{a}}$ & $0.22^{\mathrm{a}}$ & $0.22^{\mathrm{a}}$ & $0.20^{\mathrm{a}}$ \\
\hline \multirow[t]{2}{*}{3} & $8.70 \pm$ & $8.85 \pm$ & $8.95 \pm$ & $9.00 \pm$ & $9.10 \pm$ & $9.35 \pm$ & $9.40 \pm$ & $9.40 \pm$ \\
\hline & $0.20^{\mathrm{b}}$ & $0.21^{\mathrm{ab}}$ & $0.22^{\mathrm{a}}$ & $0.19^{\mathrm{a}}$ & $0.21^{\mathrm{b}}$ & $0.27^{\mathrm{a}}$ & $0.20^{\mathrm{a}}$ & $0.19^{\mathrm{a}}$ \\
\hline \multirow[t]{2}{*}{6} & $8.66 \pm$ & $8.75 \pm$ & $8.83 \pm$ & $8.85 \pm$ & $8.75 \pm$ & $9.05 \pm$ & $9.15 \pm$ & $9.34 \pm$ \\
\hline & $0.19^{b}$ & $0.18^{\mathrm{ab}}$ & $0.17^{\mathrm{a}}$ & $0.18^{\mathrm{a}}$ & $0.18^{\mathrm{b}}$ & $0.19^{\mathrm{ab}}$ & $0.21^{\mathrm{a}}$ & $0.15^{\mathrm{a}}$ \\
\hline \multirow[t]{2}{*}{9} & $8.31 \pm$ & $8.65 \pm$ & $8.75 \pm$ & $8.90 \pm$ & $8.40 \pm$ & $8.75 \pm$ & $8.85 \pm$ & $9.25 \pm$ \\
\hline & $0.18^{\mathrm{b}}$ & $0.16^{\mathrm{ab}}$ & $0.15^{\mathrm{a}}$ & $0.16^{\mathrm{a}}$ & $0.27^{\mathrm{b}}$ & $0.17^{\mathrm{ab}}$ & $0.13^{\mathrm{ab}}$ & $0.19^{\mathrm{a}}$ \\
\hline \multirow[t]{2}{*}{12} & $7.90 \pm$ & $8.35 \pm$ & $8.60 \pm$ & $8.85 \pm$ & $8.25 \pm$ & $8.41 \pm$ & $8.50 \pm$ & $9.00 \pm$ \\
\hline & $0.20^{\mathrm{bc}}$ & $0.19^{\mathrm{b}}$ & $0.16^{\mathrm{ab}}$ & $0.15^{\mathrm{a}}$ & $0.21^{\mathrm{bc}}$ & $0.19 b$ & $0.19^{\mathrm{b}}$ & $0.12^{\mathrm{a}}$ \\
\hline \multirow[t]{2}{*}{15} & $7.56 \pm$ & $7.86 \pm$ & $8.45 \pm$ & $8.70 \pm$ & $7.71 \pm$ & $8.10 \pm$ & $8.35 \pm$ & $8.75 \pm$ \\
\hline & $0.15^{\mathrm{c}}$ & $0.12^{\mathrm{bc}}$ & $0.19^{\mathrm{ab}}$ & $0.17^{\mathrm{a}}$ & $0.18^{\mathrm{bc}}$ & $0.15^{\mathrm{b}}$ & $0.17^{\mathrm{b}}$ & 0.19a \\
\hline \multirow[t]{2}{*}{18} & $7.15 \pm$ & $7.55 \pm$ & $8.16 \pm$ & $8.58 \pm$ & $7.20 \pm$ & $7.68 \pm$ & $7.86 \pm$ & $8.35 \pm$ \\
\hline & $0.17^{\mathrm{d}}$ & $0.14^{\mathrm{c}}$ & $0.16^{\mathrm{b}}$ & $0.11^{\mathrm{a}}$ & $0.18^{\mathrm{c}}$ & $0.20^{\mathrm{b}}$ & $0.19^{\mathrm{b}}$ & $0.16^{\mathrm{a}}$ \\
\hline \multirow[t]{2}{*}{21} & $6.75 \pm$ & $7.24 \pm$ & $7.80 \pm$ & $8.00 \pm$ & $6.52 \pm$ & $7.20 \pm$ & $7.42 \pm$ & $8.05 \pm$ \\
\hline & $0.19^{\mathrm{d}}$ & $0.19^{c}$ & $0.22^{\mathrm{ab}}$ & $0.14^{\mathrm{a}}$ & $0.12^{\mathrm{cd}}$ & $0.19^{\mathrm{bc}}$ & $0.22^{\mathrm{b}}$ & $0.18^{\mathrm{a}}$ \\
\hline \multirow[t]{2}{*}{24} & $6.25 \pm$ & $6.65 \pm$ & $7.50 \pm$ & $7.88 \pm$ & $6.00 \pm$ & $6.65 \pm$ & $7.01 \pm$ & $7.74 \pm$ \\
\hline & $0.18 \mathrm{~d}$ & $0.18^{\mathrm{c}}$ & $0.19^{\mathrm{b}}$ & $0.13^{\mathrm{a}}$ & $0.13^{\mathrm{cd}}$ & $0.18^{\mathrm{bc}}$ & $0.19^{\mathrm{b}}$ & $0.20^{\mathrm{a}}$ \\
\hline \multirow[t]{2}{*}{27} & $5.68 \pm$ & $6.25 \pm$ & $7.00 \pm$ & $7.60 \pm$ & $5.60 \pm$ & $5.88 \pm$ & $6.57 \pm$ & $7.35 \pm$ \\
\hline & $0.12^{\mathrm{d}}$ & $0.22^{\mathrm{c}}$ & $0.13^{\mathrm{b}}$ & $0.16^{\mathrm{a}}$ & $0.10^{\text {cd }}$ & $0.19^{c}$ & $0.17^{\mathrm{b}}$ & $0.11^{\mathrm{a}}$ \\
\hline \multirow[t]{2}{*}{30} & $5.00 \pm$ & $5.58 \pm$ & $6.35 \pm$ & $6.85 \pm$ & $5.15 \pm$ & $5.50 \pm$ & $6.20 \pm$ & $7.00 \pm$ \\
\hline & $0.10^{\mathrm{d}}$ & $0.19^{c}$ & $0.16^{\mathrm{b}}$ & $0.19^{\mathrm{a}}$ & $0.16^{\mathrm{d}}$ & $0.15^{\mathrm{c}}$ & $0.17^{\mathrm{b}}$ & $0.18^{\mathrm{a}}$ \\
\hline
\end{tabular}

${ }^{\mathrm{a}-\mathrm{d}}$ Means within a raw with different superscript are significantly different $(\mathrm{P}<0.05)$.

Values are expressed as Mean $\pm \mathrm{SD}$. 
The scores of taste and texture were significantly decreased $(\mathrm{p}<0.05)$ during storage period for all treatments. Taste scores were 9.0, for all samples at zero time of storage period, while at the end of storage period were $5,5.58$, 6.35 and 6.85, respectively. Texture scores were 9.50 for all samples at zero time of storage period, while at the end of storage period were 5.15, 5.50, 6.20 and 7.00 for control, 1,3 and $5 \%$ STPP treated samples, respectively. The highest scores of taste and texture were observed for samples treated with phosphate, while the lowest score was in control sample. The sensory properties of smoked fillets significantly decreased at the end of cold storage period. Such decrease was explained by the decrease of moisture content. The obtained data are in accordance with those reported by Suleiman (1993) and Goulas and Kontominos (2005) and Bilgin et al. (2008).

\section{Conclusion}

From the obtained results, it could be concluded that, catfish fillets treated with 5\% sodium tripolyphosphate (STPP) for $10 \mathrm{~min}$., cold smoked, packaged in polyethylene bags and stored at $4 \pm 1^{\circ} \mathrm{C}$ for 30 days are the best conditions for the product quality as long as possible since the chemical composition, physical properties and microbiological characteristic changes were less, as well as organoleptic properties scores were not significantly decreased during storage periods.

\section{REFERENCES}

Abd El-Halim, A.A. (2003). Studies on some fish oils. Ph.D. Thesis, Fac. Agric., Zagazig Univ., Egypt.

Alcicek, Z. and H.H. Atar (2010). The effects of salting on chemical quality of vacuum packed liquid smoked and traditional smoked rainbow trout (Oncorhyncus mykiss) fillets during chilled storage. J. Anim. and Vet. Adv., 9: 2778-2783.

AOAC (2005). Official Methods of Analysis of the Association of the Official Analytical Chemists, $18^{\text {th }}$ Ed. Gaithersburg, Maryland, USA.

APHA (1992). American Public Health Association, Compendium of Methods for the Microbiological Examination of Foods, $3^{\text {rd }}$ Ed., Washington, DC.

Atef, E.E., I.A. Hasanin and M.I. Salama (2008). Assessment of nutritional value of surimi and shrimp-analogues processed from catfish flesh (Clarias gariepinus L.). Abbassa Int. J. Aqua., 1 (1): 321- 336.

Bilgin, S., M. Unlusayin, L. Izci and A. Gunlu (2008). The determination of the shelf life and some nutritional components of gilthead seabream (Sparus aurata L., 1758) after cold and hot smoking. Turkish J. Vet. and Anim. Sci., 32 (1): 49-56.

Bosworth, B.G., B.C. Small and C.C. Mischke (2004). Effects of transport water temperature, aerator type, and oxygen level on channel catfish (Ictalurus punctuates) fillet quality. J. World Aquatic Soc., 35: 410417.

Duncan, D.B. (1955). Multiple Range and F test. Biometrics, 11: 1-42.

Egbal, O.A., T.A. Hawa and E.M. Kalthom (2013). Investigating the quality changes of hot smoked (Clarias lazera) at refrigerated temperature $\left(5 \pm 1^{\circ} \mathrm{C}\right)$. J. Agric. and Food Sci., 1 (3): 27-32.

El-Akeel, A.T. (1988). Chemical, microbiological and sensory evaluation of hot smoked catfish. Ph.D. Thesis. Fac. Agric., Cairo Univ., Egypt.

Gouda, H.M. (2002). Microbiological studies on some fish aquacultures in Egypt. M.Sc. Thesis, Fac. Agric., Cairo Univ., Egypt.

Goulas, A.E. and M.G. Kontominos (2005). Effect of salting and smoking method on the keeping quality of chub mackerel (Scomber japonicus): biochemical and sensory attributes. Food Chem., 93: 511-520.

Guillen, M.D. and M.C. Errecalde (2002). Volatile components of raw and smoked black bream (Brama raii) and rainbow trout (Oncorhynchus mykiss) studied by means of solid phase microextraction and gas chromatography/mass spectrometry. J. Sci. Food and Agric., 82: 945-952.

Ibrahim, S.M. (1999). Application of HACCP system in controlling hazards of some fish products. Ph.D. Thesis, Fac. Agric, AinShams Univ., Egypt. 
Kim, C.R. and D.L. Marshall (1999). Microbiological and sensory changes of refrigerated chicken legs treated with selected phosphates. Food Res. Int., 32: 209-215.

Kumolu-Johnson, C.A., N.F. Aladetohun and P.E. Ndimele (2010). The effects of smoking on the nutrional qualities and shelf-life of Clarias gariepinus (BURCHELL 1822). Afri. J. Biotechnol., 9: 073-076.

Leroi, F. and J.J. Joffraud (2000). Salt and smoke simultaneously affect chemical and sensory quality of cold-smoked salmon during $5^{\circ} \mathrm{C}$ storage predicted using factorial design. J. Food Prot., 63: 1222-1227.

Malle, P. and S.H. Tao (1986). Rapid quantitative determination of trimethylamine using steam distillation. J. Food Prot., 50: 756-760.

Moawad, R.K., M.S. Ashour, G.F. Mohamed and M.A. El-Hamzy (2013). Effect of food grade trisodium phosphate or water dip treatments on some quality attributes of decapitated white marine shrimp (Penaeus spp.) during frozen storage J. Appl. Sci. Res., 9 (6): 3723-3734.

Nahid, M.N., G.A. Latifa, F.B. Farid and M. Begum (2014). Evaluation of biochemical composition of salt and garlic treated smokedried chapila (Gudusia chapra Hamilton, 1822) and kaika (Xenentodon canc ila Hamilton-Buchanan, 1822) fish at laboratory conditions $\left(27-31^{\circ} \mathrm{C}\right)$. Res. J. Anim., Vet. and Fishery Sci., 2 (10): 10-15.

Olayemi, F.F., M.R. Adedayo, E.I. Bamishaiye and E.F. Awagu (2011). Proximate composition of catfish (Clarias gariepinus) smoked in Nigerian stored products research institute (NSPRI): Developed kiln. Int. J. Fisheries and Aquac., 3 (5): 95-97.

Özogul, Y., G. Ozyurt, F. Ozogul, E. Kuley and A. Polat (2005). Freshness assessment of European eel (Anguilla anguilla) by sensory, chemical and microbiological methods. Food Chem., 92: 745-751.

Saber, N.M., N.M. El-Shemi and S.M. Kotit (1992). Quality attributes of smoked fish products locally produced in Alexandria. J. Agric. Sci., Mansora Univ., 17: 838-846.
Saeed, S. and N. Howell (2002). Effect of lipid oxidation and frozen storage on muscle proteins of Atlantic mackerel (Scomber scombrus). J. Sci. Food and Agric., 82: 579-586.

Said El-Din, A.A., M.A. Sherif, H.A. Hashem and S.A. Abdel-Mageed (1996). Effect of smoking process and storage on quality of smoked silver carp fillets. Menofiya J. Agric. Res., 21: 355-368.

Serkan, K., K. Sevim and T. Bekir (2009). Investigating the quality changes of raw and hot smoked garfish (Belone belone euxin) at ambient and refrigerated temperatures. Turkish J. Fisheries and Aquatic Sci., 9:53-58.

Statsoft (1994). Statistica for macintosh. Statsoft and its licensors. Tulsa, OK.USA.

Sulieman, A.M. (1993). Technological studies on fish smoked keshr bayad. M.Sc. Thesis, Fac. Agric., Zagazig Univ., Egypt.

Swanson, K.M., F.F. Busta, E.H. Peterson and M.G. Johnson (1992). Colony count methods, In C. Vanderzant and D.F. Splittoesser (eds.). Compendium of methods for the microbiological examination of foods, $3^{\text {rd }} \mathrm{Ed}$. Ame. Public Health Ass., Washington, DC., 75-95.

Tarladgis, B.G., B.M. Watts, M.I. Younathan and I. Dugan (1960). A distillation method for the quantitative determination of malonaldhyde in rancid foods. J. Ame. Oil Chem. Sci., 37: 44.

Teeny, F.M. and D. Miyauchi (1972). Preparation and utilization of frozen block of minced black Reckfish muscle. J. Milk Fd. Technol., 35 (7): 414-417.

Thorarinsdottir, K.A., G. Gudmundsdottir, S. Arason, G. Thorkelsson and K. Kristbergsson (2004). Effects of added salt, phosphates and proteins on the chemical and physicochemical characteristics of frozen cod (Gadus morhua) fillets. J. Food Sci., 69 (4): 144-152.

Turan, H., Y. Kaya and I. Erkoyuncu (2003). Effects of glazing, packaging and phosphate treatments on drip loss in rainbow trout (Oncorhynchus mykiss W., 1792) during frozen storage. Turkish J. Fisheries and Aquatic Sci., 3: 105-109.

Yanar, Y. (2007). Quality changes of hot smoked catfish (Clarias gariepinus) during refrigerated storage. J. Muscle Foods, 18: 391-400. 
تحسين خواص الجودة لثرائح القراميط (Clarias gariepinus L.) المدخنة على البارد والمعاملة بالفوسفات خلال التخزين بالتبريد

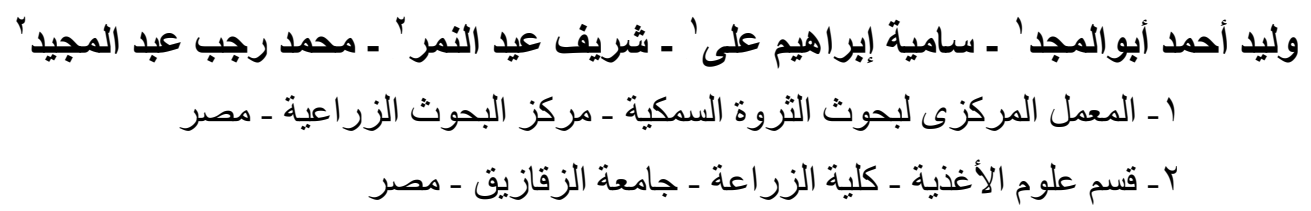

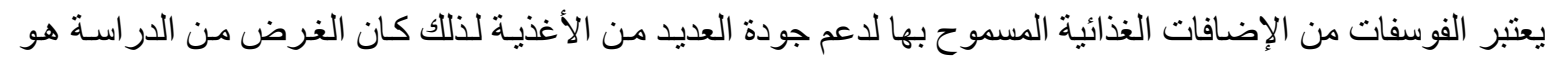

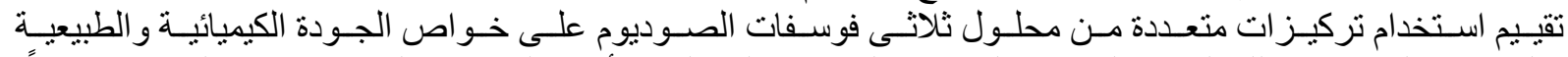

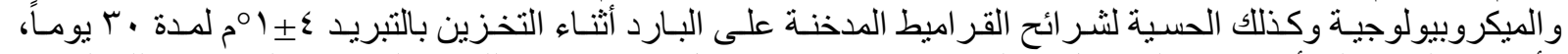

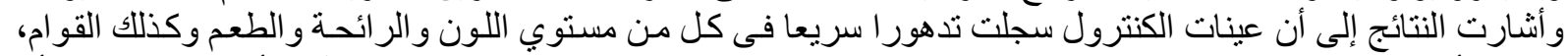

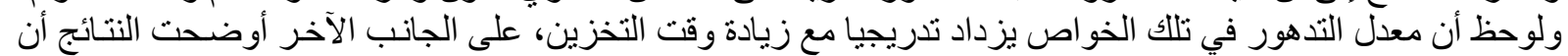

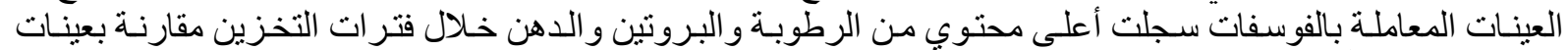

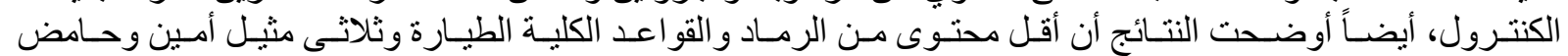

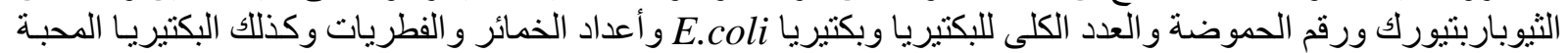

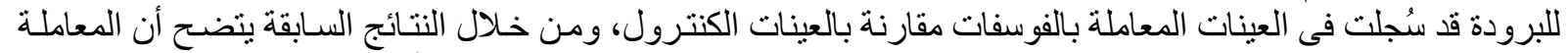

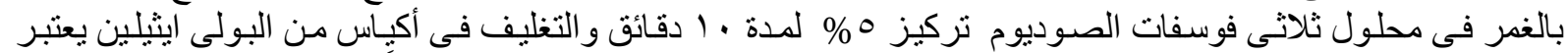

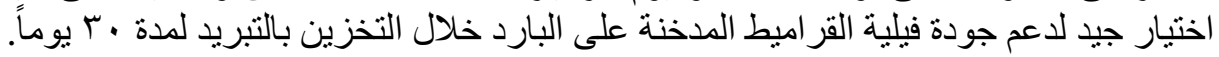

أستاذ الصناعات الغذائية ـ كلية التكنولوجيا و التنمية - جامعة الزقازيق.

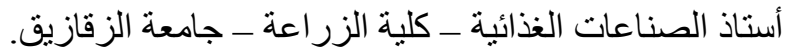

